\title{
Consumo de sódio e potássio por gestantes do Vale do Jequitinhonha
}

\author{
Sodium and potassium intake by pregnant women from Vale do Jequitinhonha
}

\author{
Humberto Gabriel Rodriguesa, Jéssica Carvalho Freitas ${ }^{b}$, Lucianne Vanelle Sales Freitas ${ }^{b}$, \\ Karide Christiane Ladeia Sena ${ }^{b}$ \\ a Biólogo. Doutor em Ciências da Saúde pela Universidade de Brasília (UNB). Professor do Curso de Medicina das Faculdades Integradas Pitágoras de Montes Claros e da \\ Universidade Estadual de Montes Claros, Montes Claros, MG, Brasil. \\ b Acadêmica de Medicina das Faculdades Integradas Pitágoras de Montes Claros, Montes Claros, MG, Brasil.
}

RESUMO

Introdução: Durante a gestação, as necessidades quanto aos nutrientes tendem a aumentar com a finalidade de que haja um aporte preciso à manutenção do metabolismo da gestante e ao desenvolvimento do feto. Tem-se comprovado, por exemplo, a influência do sódio e do potássio na regulação da pressão arterial, o que é de extrema importância durante a gestação.

Objetivo: Avaliar a ingestão dos micronutrientes sódio e potássio nas gestantes do Vale do Jequitinhonha (Minas Gerais, Brasil). Frente ao fato de que essa região é considerada pelos parâmetros da UNESCO (Organização das Nações Unidas para a Educação a Ciência e a Cultura) uma das mais pobres do Brasil, esse se torna um local oportuno para a realização do estudo.

Materiais e Métodos: Trata-se de um estudo transversal. A pesquisa foi realizada em 492 gestantes atendidas em maternidades de 15 municípios do Vale do Jequitinhonha, nas quais são realizados $78 \%$ dos partos desta região. Para a coleta de dados, foi utilizado um questionário padronizado que inclui um inquérito de consumo alimentar, além de um questionário para verificação da situação socioeconômica das gestantes.

Resultados: Observou-se que o total de mulheres com ingestão de sódio abaixo de Al (ingestão adequada) foi de 35,03\%, acima de UL, 46,43\% e, de potássio, a ingestão abaixo de Al ocorreu em 148 mulheres (30,14\%).

Conclusão: Verificou-se inadequação no consumo de sódio e potássio pelas gestantes analisadas, o que pode refletir em alterações nos níveis pressóricos maternos e no desenvolvimento fetal.

Palavras-chave: consumo de sódio; consumo de potássio; gestantes; Brasil.

ABSTRACT

Introduction: During pregnancy, the needs for nutrients tend to increase with the purpose that there is a clear contribution to metabolism of the pregnant woman maintenance and development of the fetus. It has been proven, for example, the influence of sodium and potassium in blood pressure regulation, which is extremely important during pregnancy.

Objective: To evaluate the intake of sodium and potassium micronutrients in pregnant women in the Jequitinhonha Valley (Minas Gerais, Brazil). Given the fact that this region is considered by UNESCO parameters (the United Nations Educational, Scientific and Cultural Organization) one of the poorest in Brazil, this becomes a suitable place for the study.

Materials and Methods: This is a cross-sectional study. The study was conducted on 492 pregnant women in maternity wards in 15 municipalities in the Jequitinhonha Valley, in which are held $78 \%$ of deliveries in this region. To collect data, we used a standardized questionnaire that includes a survey of food consumption, as well as a questionnaire to verify the socioeconomic status of pregnant women.

Results: It was observed that the total number of women with sodium intake below Al (adequate intake) was 35.03\%, up from UL, 46.43\% and potassium intake below Al occurred in 148 women (30.14\%).

Conclusion: There was inadequate consumption of sodium and potassium by the pregnant women analyzed, which may reflect changes in maternal blood pressure and fetal development.

Keywords: sodium intake; potassium intake; pregnant women; Brazil.

\section{Correspondência:}

HUMBERTO GABRIEL RODRIGUES

Departamento de Medicina, Faculdades Integradas Pitágoras de Montes Claros

Av. Professora Aida Mainartina Paraiso, 80 - Ibituruna

39408-007 Montes Claros, MG, Brasil

E-mail: humbertobriel@gmail.com 


\section{INTRODUÇÃO}

A alimentação tem papel expressivo para a saúde dos indivíduos, singularmente nos períodos da vida caracterizados por uma maior demanda de energia e de nutrientes, como a gestação. Nessa etapa, acontece profundo e intrínseco processo de formação de tecidos e grandes modificações orgânicas durante um pequeno espaço de tempo ${ }^{1}$.

Durante a gestação ocorrem diversas modificações fisiológicas, tendo essas uma participação efetiva nas necessidades nutricionais. Percebe-se então que, para a gravidez ter o êxito esperado, é essencial o atendimento à demanda nutricional durante esse período a fim de propiciar um satisfatório ganho ponderal gestacional e bom desfecho obstétrico definido pelo perfeito desenvolvimento do feto e nascimento do bebê em idade gestacional adequada ${ }^{2}$.

Dentre as necessidades nutricionais ocorre um aumento na requisição de micronutrientes com o objetivo de manter o organismo materno com reservas apropriadas e condicionar o desenvolvimento fetal ${ }^{2}$. Tem-se, por exemplo, o sódio e o potássio que são minerais essenciais à regulação dos fluidos intra e extracelulares, atuando na manutenção da pressão sanguínea. O cloreto de sódio é constituído por $40 \%$ de sódio, estando à principal fonte desse mineral na alimentação ${ }^{3}$.

O desequilíbrio desses minerais pode causar edema, um processo considerado comum no período gestacional; no entanto, inspira cuidados e, dessa forma, a ingestão desses minerais, principalmente o sódio, deve correr de forma controlada. Lembrando-se que a redução da ingestão de sódio deve ser realizada apenas em situações específicas ${ }^{4}$.

Além disso, segundo Sacks et al. ${ }^{5}$, mesmo que os meios de atuação dos fatores nutricionais sobre a ascendência da pressão arterial ainda não estejam bem elucidados, são conhecidas consequências de uma dieta saudável sobre o desempenho dos níveis pressóricos. Dentre os diversos fatores nutricionais já estudados, sabe-se que a ingestão exagerada de sódio é um dos principais fatores de risco para a hipertensão arterial sistêmica ${ }^{6}$.

Atualmente, sugere-se que a ingestão até $1,7 \mathrm{~g}$ de sódio (5g de cloreto de sódio por dia) possa auxiliar na redução da pressão arterial. Observa-se que grande parte das pessoas, mesmo as crianças, consome níveis desse nutriente além de suas necessidades. O consumo populacional excessivo, maior que $6 \mathrm{~g}$ diárias (2,4g de sódio), é uma justificativa importante ao surgimento da hipertensão arterial ${ }^{4}$.

Há relatos de que a hipertensão gestacional é a complicação mais comum na gestação sendo, pois, a principal causa de mortalidade. Esse distúrbio alcança todas as idades e raças, ocorrendo nas gestantes com menos de 20 anos e mais de 40 anos $^{7}$. Quando a doença hipertensiva atinge as mulheres durante a gravidez, são chamadas de Pré-Eclâmpsia e Eclâmpsia, sendo essas complicações graves problemas de saúde públicaㄱ. Isso é devido ao fato de que, além do risco de morte para a gestante, essas complicações também são responsáveis por consequências aos neonatos, como por exemplo, a encefalopatia neonatal ${ }^{8}$. Frente a isso, dentre os cuidados requeridos estão o repouso e a diminuição da ingestão de sal ${ }^{7}$.

Cerca de $10 \%$ das gestantes são afetadas por hipertensão arterial sistêmica, sendo inserida entre as causas predominantes de internação, morbimortalidade materna e perinatal, inclusive no Brasil $^{10}$. Esse fato demonstra a importância na regulação nutricional dessas mulheres nessa importante fase da vida intrauterina. Quando a gestante tem um quadro de desnutrição, estudos apontam que há a probabilidade de nascer uma criança com baixo peso, ${ }^{11} \mathrm{com}$ deficiências nutricionais, retardo mental e comprometimento do crescimento intrauterino, entre outros fatores ${ }^{12}$.

Tendo em vista que a dieta equilibrada é de extrema relevância, pois tanto a restrição como o excesso de determinados nutrientes no período gestacional pode interferir no desenvolvimento e crescimento do feto, o presente trabalho tem por objetivo estimar a ingestão dos minerais sódio e potássio por gestantes do Vale do Jequitinhonha, uma das regiões mais pobres do Brasil.

\section{MATERIAIS E MÉTODOS}

Trata-se de um estudo transversal de caráter descritivo e uma abordagem quantitativa entre gestantes assistidas em unidades básicas de saúde de quinze municípios do Vale do Jequitinhonha. O cenário estudado foi Vale do Jequitinhonha, que é uma região que ocupa 14,5\% da área do Estado de Minas Gerais, totalizando aproximadamente $85.000 \mathrm{Km}^{2}$ de extensão territorial, situado geograficamente no Nordeste de Minas Gerias, fazendo fronteiras com o Mucuri e o Norte de Minas. Segundo pesquisas do Sistema de Informações hospitalares do Sistema Único de Saúde (SIH/SUS) do Ministério da Saúde realizada em 2010, o Vale do Jequitinhonha possui oitenta municípios e, destes, vinte e nove possuem registros de parto.

A amostragem para coleta de dados primários foi obtida contemplando como universo da pesquisa o número total de partos no Vale do Jequitinhonha, MG. No ano de 2010, ocorreram aproximadamente 12.500 partos, o que, consequentemente, representa o número de gestantes dessa macrorregião ${ }^{13}$. A investigação em campo foi realizada de janeiro de 2013 a julho de 2014, com 492 gestantes em unidades básicas de saúde (UBSs) de 15 dos 29 municípios selecionados devido ao critério de realização de pelo menos 
um parto por dia, perfazendo o total de 30 partos ao mês ou que fossem realizados $78 \%$ dos partos na região. Duas a três unidades de saúde foram selecionadas para cada município onde a pesquisa foi realizada.

Foi aplicado inquérito alimentar entre as gestantes através da aplicação de questionário padronizado de Frequência Alimentar Semiquantitativo para quantificação dos micronutrientes sódio e potássio, questionário estabelecido como padrão, antecipadamente testado por mediação de estudo-piloto, preenchido durante a entrevista, após a obtenção do consentimento da mulher e empregado à coleta de dados.

De acordo com Padovani et al. ${ }^{14} \mathrm{o}$ UL (limite superior de maior ingestão de um nutriente) para o sódio é 2,3 gramas em gestantes. Porém, neste estudo, o valor de UL para o potássio não pôde ser estabelecido. No caso do Al (ingestão adequada) foi de 1,5 gramas para sódio e de 4,7 gramas para potássio.

Entrevistadores instruídos aplicaram os questionários, sendo que os mesmos foram acompanhados por pesquisador mais experiente em pesquisa de campo. Foi aplicado também questionário sociodemográfico incluído as variáveis: cor da pele (auto referida); a situação conjugal da mulher (com companheiro e sem companheiro); renda familiar (em reais); participação no Programa Bolsa Família; escolaridade (em anos); e idade (em anos completos) da mãe. Como variável de conduta, foi pesquisada, também, a gestação planejada (não/sim); gravidez na adolescência (sim para menor de 18 anos) e número de gestações. As variáveis do pré-natal consistem na realização do mesmo (não/sim), número de consultas e local de ocorrência destas e se a gestante recebeu orientações nutricionais durante esse período; caso positivo, por quem foram realizadas tais orientações, motivo e período da mesma.

Dentre todos os alimentos pesquisados através do questionário padronizado de Frequência Alimentar Semiquantitativo com 144 alimentos; 141 alimentos possuem alguma quantidade de sódio em sua composição. No caso do potássio, todos os alimentos utilizados na pesquisa possuem potássio. Sendo assim, na análise da quantidade de sódio presente nos produtos, três alimentos foram excluídos por não conterem esse micronutriente.

As gestantes incluídas na pesquisa estavam no terceiro trimestre de gestação no momento da aplicação do questionário, habitavam em um dos oitenta Municípios do Vale do Jequitinhonha e consentiram a participar da pesquisa através da assinatura do Termo de Consentimento Livre e Esclarecido. O projeto foi submetido ao Comitê de Ética da Universidade Estadual de Montes Claros (UNIMONTES) e aprovado sob o protocolo número 3094.
Os dados do questionário foram digitados no Excel e, posteriormente, transferidos para o Epiinfo (42) para a análise estatística. No caso das variáveis quantitativas contínuas, como idade e consumo alimentar, calcularam-se as médias, medianas e desvio padrão. Para as variáveis discretas foram calculadas frequências, mediana, desvio padrão, intervalos de confiança (95\%) e, para os testes de associação, a razão de prevalência (RP), considerando-se o limite de confiança de $95 \%$.

\section{RESULTADOS}

Como relatado na metodologia deste estudo, 492 mulheres atendidas nas unidades básicas de saúde no Vale do Jequitinhonha participaram da pesquisa. Destas, $66,7 \%$ tinham um salário mínimo que constituía o sustento da família e 45,7\% têm o benefício do "Bolsa Família" (Tabela 1).

Dentre as entrevistadas, observou-se na idade uma média de 25,3, sendo os extremos 13 e 43 anos. A etnia prevalente foi morena ou parda, ocupando $78,6 \%$ das gestantes e as que se declararam amarelas, apenas 3,9\%. Trezentas e cinquenta e seis $(72,3 \%)$ mulheres afirmaram ter um companheiro; $27,6 \%$ afirmaram não ter e 01 não respondeu a questão. A maioria das entrevistadas $(59,7 \%)$ tinha o ensino médio completo ou incompleto e apenas $33(6,7 \%)$ tinham o ensino superior completo ou ainda não haviam concluído o mesmo (Tabela 1).

Nesta pesquisa, a maior parte $(39,8 \%)$ das mulheres estavam gestando pela primeira vez; $56,7 \%$ não haviam planejado a gestação e $66,1 \%$ participaram de menos do que seis consultas pré-natal. Durante a gravidez 3,9\% das mulheres afirmaram ter o ato de fumar e 7,3\% relataram ter a prática do tabagismo antes da gestação. Observou-se que 297 gestantes não receberam orientação alimentar durante a gestação, número equivalente a $60,4 \%$. Aproximadamente $40,0 \%$ das mulheres $(n=195)$ foram orientadas em relação à nutrição por profissionais da saúde, 150 (76,3\%) destas, durante a gravidez. $\mathrm{O}$ total de mulheres com ingestão de sódio abaixo de Al foi de 35,03\%, acima de UL, 46,43\%. Já em relação ao potássio, a ingestão abaixo de $\mathrm{Al}$ foi realizada por 148 mulheres, correspondendo a 30,14\% (Tabela 1).

Analisando-se os fatores (Tabela 2), tem-se que uma parte deles correlacionou-se com uma ingesta insuficiente de sódio, como a não inserção no projeto governamental Bolsa Família $(R P=1,16)$, escolaridade cursada até o nível médio ( $R P=1,31)$, menos do que seis consultas de pré-natal $(R P=1,28)$ e mais de três refeições por dia $(R P=1,52)$.

Através da Tabela 3, podemos identificar o fator relacionado a um consumo excessivo de sódio, apenas em mulheres sem companheiro $(\mathrm{RP}=1,32)$. 
Dentre os fatores identificados, alguns se correlacionaram com a baixa ingesta de potássio (Tabela 4). Estes foram à etnia amarela $(\mathrm{RP}=2,41)$, no estado civil sem um companheiro
$(R P=1,48)$, número de consultas menor do que seis $(R P=1,41)$ e o número de refeições por dia maior do que três $(R P=1,59)$.

Tabela 1. Características das gestantes atendidas pelo Sistema Único de Saúde, incluídas na amostra.

\begin{tabular}{|c|c|c|c|}
\hline Característica & Categoria & $\begin{array}{c}\text { № de } \\
\text { gestantes }\end{array}$ & $\begin{array}{c}\text { Porcentagem } \\
\text { (\%) }\end{array}$ \\
\hline \multirow[t]{4}{*}{ Idade (anos) } & $10-19$ & 100 & 20,3 \\
\hline & $20-34$ & 346 & 70,3 \\
\hline & $35-45$ & 43 & 8,8 \\
\hline & Não responderam & 3 & 0,6 \\
\hline \multirow[t]{3}{*}{ Etnia $^{a}$ (autodeclarada) } & Amarela & 19 & 3,9 \\
\hline & Branca & 86 & 17,5 \\
\hline & Negra ou parda & 387 & 78,6 \\
\hline \multirow[t]{3}{*}{ Estado Civil ${ }^{\mathrm{a}}$} & Com companheiro & 356 & 72,3 \\
\hline & Sem companheiro & 136 & 27,6 \\
\hline & Não responderam & 1 & 0,3 \\
\hline \multirow[t]{4}{*}{ Escolaridade $^{\mathrm{a}}$} & Fundamental & 161 & 32,7 \\
\hline & Médio & 294 & 59,7 \\
\hline & Superior & 33 & 6,7 \\
\hline & Não responderam & 4 & 0,8 \\
\hline \multirow[t]{2}{*}{ Renda familiara } & Até um salário mínimo & 328 & 66,7 \\
\hline & Mais um salário mínimo & 164 & 33,3 \\
\hline \multirow[t]{2}{*}{ Participação Bolsa Famíliaa } & Sim & 225 & 45,7 \\
\hline & Não & 267 & 54,3 \\
\hline \multirow[t]{4}{*}{ Número de gestações ${ }^{a}$} & Uma & 196 & 39,8 \\
\hline & Duas & 147 & 29,9 \\
\hline & Três & 89 & 18,1 \\
\hline & Acima de três & 60 & 12,2 \\
\hline \multirow[t]{2}{*}{ Número de consultas de pré-natala } & Menos do que seis & 325 & 66,1 \\
\hline & Seis ou mais & 167 & 33,9 \\
\hline \multirow[t]{3}{*}{ Planejamento da gravidez ${ }^{a}$} & Sim foi planejada & 210 & 42,7 \\
\hline & Não foi planejada & 279 & 56,7 \\
\hline & Não responderam & 3 & 0,6 \\
\hline \multirow[t]{2}{*}{ Recebeu orientação alimentar durante a gestação ${ }^{a}$} & Sim & 195 & 39,6 \\
\hline & Não & 297 & 60,4 \\
\hline \multirow[t]{2}{*}{ Tabagismo antes da gestação ${ }^{a}$} & Sim & 36 & 7,3 \\
\hline & Não & 456 & 92,7 \\
\hline \multirow[t]{2}{*}{ Tabagismo durante gestação ${ }^{a}$} & Sim & 19 & 3,9 \\
\hline & Não & 473 & 96,1 \\
\hline \multirow[t]{2}{*}{ Consumo de sódio (total Al) } & Suficiente & 172 & 35,03 \\
\hline & Inadequado & 319 & 64,97 \\
\hline \multirow[t]{2}{*}{ Consumo de sódio (total UI) } & Suficiente & 263 & 53,56 \\
\hline & Excedente & 228 & 46,43 \\
\hline \multirow[t]{2}{*}{ Consumo de potássio (total $\mathrm{Al}$ ) } & Suficiente & 148 & 30,14 \\
\hline & Inadequado & 343 & 69,86 \\
\hline
\end{tabular}

a Tamanho da amostra $(\mathrm{n}=492)$. 
Tabela 2. Prevalência de consumo insuficiente de sódio segundo características econômicas e gestacionais.

\begin{tabular}{|c|c|c|c|c|c|c|}
\hline Característica & $\begin{array}{c}\text { № de } \\
\text { gestantes }\end{array}$ & $\mathrm{Al}<1,5$ & $\mathrm{Al}>1,5$ & $\begin{array}{c}\text { Consumo } \\
\text { inadequado } \\
(\%)\end{array}$ & $\mathbf{R P}$ & IC $95 \%$ \\
\hline \multicolumn{7}{|l|}{ Renda familiar } \\
\hline Até um salário mínimo & 328 & 116 & 212 & 64,7 & 1 & \\
\hline Mais de um salário mínimo & 164 & 57 & 107 & 65,2 & 1,0 & $0,88-1,16$ \\
\hline \multicolumn{7}{|l|}{ Participação no Bolsa Família } \\
\hline $\operatorname{Sim}$ & 225 & 93 & 132 & 58,7 & 1 & \\
\hline Não & 267 & 84 & 183 & 68,5 & 1,16 & $1,02-1,34$ \\
\hline \multicolumn{7}{|l|}{ Idade materna } \\
\hline 10-19 anos & 100 & 32 & 68 & 68 & 1,08 & $0,92-1,26$ \\
\hline 20-34 anos & 346 & 128 & 218 & 63 & 1 & \\
\hline $35-44$ anos & 43 & 12 & 31 & 72,1 & 1,14 & $0,93-1,40$ \\
\hline \multicolumn{7}{|l|}{ Etnia } \\
\hline Branca & 86 & 40 & 46 & 53,5 & 1,02 & $0,63-1,62$ \\
\hline Negra/ Parda & 387 & 124 & 263 & 68 & 1,29 & $0,84-1,99$ \\
\hline Amarela & 19 & 9 & 10 & 52,6 & 1 & \\
\hline \multicolumn{7}{|l|}{ Estado Civil } \\
\hline Com companheiro & 356 & 131 & 225 & 63,2 & 1 & \\
\hline Sem companheiro & 136 & 42 & 94 & 69,1 & 1,09 & $0,95-1,25$ \\
\hline \multicolumn{7}{|l|}{ Escolaridade } \\
\hline Fundamental & 161 & 74 & 87 & 54,4 & 1 & \\
\hline Médio & 294 & 85 & 209 & 79,8 & 1,31 & $1,12-1,54$ \\
\hline Superior & 33 & 12 & 21 & 63,6 & 1,18 & $0,88-1,58$ \\
\hline \multicolumn{7}{|l|}{ Planejamento da gravidez } \\
\hline Não planejada & 279 & 100 & 176 & 63,8 & 1 & \\
\hline Planejada & 210 & 69 & 143 & 67,4 & 1,06 & $0,92-1,20$ \\
\hline \multicolumn{7}{|l|}{ Número de gestações } \\
\hline Uma & 196 & 67 & 129 & 65,8 & 1,03 & $0,90-1,18$ \\
\hline Acima de uma & 296 & 107 & 189 & 63,8 & 1 & \\
\hline \multicolumn{7}{|l|}{ Número de consultas pré-natal } \\
\hline Menor que seis & 325 & 84 & 218 & 67,1 & 1,28 & $1,10-1,49$ \\
\hline Igual ou maior que seis & 167 & 73 & 94 & 56,3 & 1 & \\
\hline \multicolumn{7}{|l|}{ Número de refeições por dia } \\
\hline Até três & & 76 & 67 & 46,8 & 1 & \\
\hline Mais de três & & 100 & 247 & 71,2 & 1,52 & $1,26-1,83$ \\
\hline
\end{tabular}

Al: ingestão insuficiente; RP: razão de prevalência; IC: intervalo de confiança. 
Tabela 3. Prevalência de consumo de sódio em relação ao que é tolerável (UL) segundo características econômicas e gestacionais.

\begin{tabular}{|c|c|c|c|c|c|c|c|}
\hline Característica & $\begin{array}{c}\text { № de } \\
\text { gestantes }\end{array}$ & $\mathrm{UI}<2,3 \mathrm{~g}$ & $\mathrm{UI}>2,3 \mathrm{~g}$ & $\begin{array}{c}\text { Consumo } \\
\text { que não excede } \\
\text { o limite (\%) }\end{array}$ & $\begin{array}{l}\text { Consumo que } \\
\text { excede o limite } \\
(\%)\end{array}$ & $\mathbf{R P}$ & IC $95 \%$ \\
\hline \multicolumn{8}{|l|}{ Renda familiar } \\
\hline Até um salário mínimo & 328 & 182 & 146 & 55,5 & 44,5 & 1 & \\
\hline Mais de um salário mínimo & 164 & 84 & 80 & 51, & 48,9 & 1,09 & $0,90-1,33$ \\
\hline \multicolumn{8}{|l|}{ Participação no Bolsa Família } \\
\hline Sim & 225 & 114 & 111 & 50,7 & 49,3 & 1,15 & $0,95-1,4$ \\
\hline Não & 267 & 153 & 114 & 57,3 & 42,7 & 1 & \\
\hline \multicolumn{8}{|l|}{ Idade materna } \\
\hline 10-19 anos & 100 & 58 & 42 & 58 & 42 & 1 & \\
\hline 20-34 anos & 346 & 184 & 162 & 53,2 & 46,3 & 1,11 & $0,86-1,44$ \\
\hline $35-44$ anos & 43 & 22 & 21 & 51,2 & 48,8 & 1,62 & $0,79-1,70$ \\
\hline \multicolumn{8}{|l|}{ Etnia } \\
\hline Branca & 86 & 55 & 31 & 63,9 & 36 & 1 & \\
\hline Negra/ Parda & 387 & 201 & 186 & 51,9 & 48,1 & 1,33 & $0,99-1,80$ \\
\hline Amarela & 19 & 9 & 10 & 10,6 & 89,4 & 1,46 & $0,97-2,43$ \\
\hline \multicolumn{8}{|l|}{ Estado Civil } \\
\hline Com companheiro & 356 & 205 & 151 & 57,6 & 42,4 & 1 & \\
\hline Sem companheiro & 136 & 60 & 76 & 44,1 & 55,9 & 1,32 & $1,09-1,60$ \\
\hline \multicolumn{8}{|l|}{ Escolaridade } \\
\hline Fundamental & 161 & 87 & 74 & 54,0 & 46 & 1 & \\
\hline Médio & 294 & 158 & 136 & 53,7 & 46,3 & 1 & $0,82-1,24$ \\
\hline Superior & 33 & 17 & 16 & 51,5 & 48,5 & 1,05 & $0,71-1,56$ \\
\hline \multicolumn{8}{|l|}{ Planejamento da gravidez } \\
\hline Não planejada & 279 & 140 & 138 & 50,3 & 49,6 & 1,21 & $0,99-1,48$ \\
\hline Planejada & 210 & 124 & 86 & 59 & 41 & 1 & \\
\hline \multicolumn{8}{|l|}{ Número de gestações } \\
\hline Uma & 196 & 106 & 90 & 54,1 & 45,9 & 1 & \\
\hline Acima de uma & 296 & 159 & 137 & 53,7 & 46,3 & 1 & $0,83-1,22$ \\
\hline \multicolumn{8}{|l|}{ Número de consultas pré-natal } \\
\hline Menor que seis & 325 & 165 & 160 & 50,8 & 49,2 & 1,23 & $0,99-1,52$ \\
\hline Igual ou maior que seis & 167 & 100 & 67 & 59,9 & 40,1 & 1 & \\
\hline \multicolumn{8}{|l|}{ Número de refeições por dia } \\
\hline Até três & & 73 & 65 & 52,9 & 47,1 & 1,03 & $0,83-1,27$ \\
\hline Mais de três & & 191 & 162 & 54,1 & 45,9 & 1 & \\
\hline
\end{tabular}

UL: limite tolerável; RP: razão de prevalência; IC: intervalo de confiança. 
Tabela 4. Prevalência de consumo insuficiente de potássio segundo características econômicas e gestacionais.

\begin{tabular}{|c|c|c|c|c|c|c|}
\hline Característica & $\begin{array}{c}\text { № de } \\
\text { gestantes }\end{array}$ & $A \mathrm{I}<4,7$ & $\mathrm{Al}>4,7$ & $\begin{array}{c}\text { Consumo } \\
\text { inadequado } \\
(\%)\end{array}$ & RP & IC $95 \%$ \\
\hline \multicolumn{7}{|l|}{ Renda familiar } \\
\hline Até um salário mínimo & 328 & 232 & 96 & 29,3 & 1,04 & $0,77-1,40$ \\
\hline Mais de um salário mínimo & 164 & 118 & 46 & 28,0 & 1 & \\
\hline \multicolumn{7}{|l|}{ Participação no Bolsa Família } \\
\hline Sim & 225 & 156 & 69 & 30,6 & 1,19 & $0,89-1,57$ \\
\hline Não & 267 & 198 & 69 & 25,8 & 1 & \\
\hline \multicolumn{7}{|l|}{ Idade materna } \\
\hline 10-19 anos & 100 & 72 & 28 & 28 & 1 & \\
\hline 20-34 anos & 346 & 243 & 103 & 29,8 & 1,06 & $0,75-1,51$ \\
\hline $35-44$ anos & 43 & 30 & 13 & 30,2 & 1,08 & $0,62-1,87$ \\
\hline \multicolumn{7}{|l|}{ Etnia } \\
\hline Branca & 86 & 71 & 15 & 17,6 & 1 & \\
\hline Negra/ Parda & 387 & 268 & 119 & 30,8 & 1,76 & $1,09-2,86$ \\
\hline Amarela & 19 & 11 & 8 & 42,1 & 2,41 & $1,20-4,86$ \\
\hline \multicolumn{7}{|l|}{ Estado Civil } \\
\hline Com companheiro & 356 & 265 & 91 & 25,6 & 1 & \\
\hline Sem companheiro & 136 & 85 & 51 & 37,5 & 1,48 & $1,11-1,94$ \\
\hline \multicolumn{7}{|l|}{ Escolaridade } \\
\hline Fundamental & 161 & 115 & 46 & 28,6 & 1,35 & $0,67-2,72$ \\
\hline Médio & 294 & 206 & 88 & 29,9 & 1,41 & $0,71-2,79$ \\
\hline Superior & 33 & 26 & 7 & 21,2 & $" 1$ & \\
\hline \multicolumn{7}{|l|}{ Planejamento da gravidez } \\
\hline Não planejada & 279 & 188 & 90 & 32,4 & 1,31 & $0,98-1,75$ \\
\hline Planejada & 210 & 158 & 52 & 24,8 & 1 & \\
\hline \multicolumn{7}{|l|}{ Número de gestações } \\
\hline Uma & 196 & 143 & 53 & 27,0 & 1 & \\
\hline Acima de uma & 296 & 207 & 89 & 30,1 & 0,9 & $0,67-1,20$ \\
\hline \multicolumn{7}{|l|}{ Número de consultas pré-natal } \\
\hline Menor que seis & 325 & 221 & 104 & 32,0 & 1,41 & $1,02-1,94$ \\
\hline Igual ou maior que seis & 167 & 129 & 38 & 22,8 & 1 & \\
\hline \multicolumn{7}{|l|}{ Número de refeições por dia } \\
\hline Até três & & 110 & 28 & 20,3 & 1 & \\
\hline Mais de três & & 239 & 114 & 32,3 & 1,59 & $1,11-2,29$ \\
\hline
\end{tabular}

Al: ingestão insuficiente; RP: razão de prevalência; IC: intervalo de confiança. 


\section{DISCUSSÃO}

Em relação à ingestão de sódio, a maioria $(64,97 \%)$ das entrevistadas apresentou inadequação na ingestão desse nutriente e 46,43\% ingeriram acima da quantidade limítrofe (UL). No primeiro caso tiveram relação com a maior ingesta, as gestantes que realizaram um menor número de consultas pré-natais, o que pode ser explicado de acordo com o trabalho ${ }^{16}$ que ressalta a importância das intervenções que podem ser realizadas durante essas consultas, como por exemplo, garantir um suporte nutricional durante a fase gestacional dessas mulheres. Observa-se então que há uma interferência direta entre as consultas e nutrição na gestação, como também na baixa ingestão de potássio. Já foi relatada a existência de uma alta proporção de mulheres em fase reprodutiva que ingere dietas com baixas quantidades de micronutrientes ${ }^{17}$.

Ao analisar-se a baixa ingesta de potássio, encontrou-se a relação com mulheres de etnia amarela. O estudo de Lacerda et al. ${ }^{17}$ constatou o fato de uma inadequação na ingesta alimentar para todas as mulheres, com elevado consumo de energia no período gestacional em mulheres pretas e pardas, o que não foi encontrado em nossa análise. Além disso, houve uma relação com ausência de um companheiro e um número maior do que três refeições por dia.

Uma pesquisa realizada no Município de São Paulo observou que as gestantes sem companheiro tiveram 2,5 vezes mais chances de ganho de peso semanal excessivo, tanto no segundo quanto no terceiro trimestre, demonstrando uma inadequação dietética nessas mulheres ${ }^{18}$. No estudo, isso foi tido como causa possível, o fato de a ausência de companheiro demonstrar um apoio psicossocial inadequado ${ }^{19}$.

A não inserção no programa governamental Bolsa Família, foi relacionado a uma baixa ingestão de sódio, assim como a escolaridade ao nível de ensino médio. A baixa ingestão de nutrientes pode ocorrer em população com menor nível escolar e com falta de apoio financeiro, como a região estudada, em que há uma quantidade significativa de famílias de baixa renda. No estudo afirma-se que essas transferências de renda auxiliam famílias que vivem em contextos desfavoráveis e cuja condição nutricional sofre impacto de diversas maneiras, tais como as dificuldades de ter de modo adequado o acesso a alimentos em quantidade e qualidade para consumo ${ }^{20}$.

No trabalho foi identificada a relação entre o consumo excessivo de sódio e mulheres sem companheiro. Essa irregularidade alimentar relacionada ao estado conjugal foi relatada afirmando-se que a presença do companheiro é algo importante para aquela que está gestando, uma vez que ser mãe solteira pode trazer pontos negativos à saúde psicológica, podendo acarretar em uma menor estabilidade econômica na família, com consequente fator de risco para o baixo peso ao nascer ${ }^{21}$.

Os resultados apresentados demonstram a necessidade de se associar às gestações aos fatores citados que interferem na qualidade nutricional da mulher, visto que estes corroboram com consequências para a criança que está sendo gerada. Isso demonstra a necessidade da instrução nutricional antes e durante a gravidez e medidas que aumenta o acesso de populações, principalmente em regiões pobres como o Vale do Jequitinhonha, a alimentos saudáveis com a quantidade correta desses micronutrientes.

Neste estudo foi possível observar que 297 gestantes não receberam orientação alimentar durante a gestação, número equivalente a $60,4 \%$. Diante do exposto sobre as complicações que podem ocorrer com uma ingestão inadequada de sódio e potássio, por exemplo, esse número torna-se considerável, prejudicando sobremaneira o bemestar materno e fetal.

Dessa forma, verificou-se inadequação no consumo de sódio e potássio pelas gestantes analisadas, o que pode refletir em alterações nos níveis pressóricos maternos e no desenvolvimento fetal. Diante disso, é facilmente observada o quanto uma ingesta alimentar adequada influencia na vida da gestante e da criança que está sendo gerada. Logo, é de suma importância a necessidade de intervenções preventivas, principalmente em regiões precárias, para que medidas paliativas não sejam necessárias.

\section{REFERÊNCIAS}

1. Coelho KS, Souza AI, Batista FM. Avaliação antropométrica do estado nutricional da gestante: visão retrospectiva e prospectiva. Rev Bras Saúde Matern Infant. 2002;2(1):57-61. https://doi. org/10.1590/s1519-38292002000100009

2. Guimarães AF, Silva SMCS. Necessidades e recomendações nutricionais na gestação. Cad Fac Integr São Camilo. 2003;9(2):36-49.

3. Ministério da Saúde (BR). Guia alimentar para a população brasileira: promovendo a alimentação saudável. Brasília: Ministério da Saúde; 2008.

4. Saunders C. Síndromes hipertensivas da gravidez - SHG. In: Accioly E, Saunders C, Lacerda EMA, organizadores. Nutrição em obstetrícia e pediatria. 2a ed. Rio de Janeiro: Cultura Médica; 2002. p. 189207.

5. Sacks FM, Svetkey LP, Vollmer WM, Appel LJ, Bray GA, Harsha D. Effects on blood pressure ofreduced dietary sodium and the Dietary Approaches to Stop Hypertension (DASH) diet. N Engl J Med. 2001;344(1):3-10. https://doi.org/10.1056/NEJM 200101043440101

6. Zhao D, QI Y, Zheng Z, Wang Y, Zhangxy LJ. Dietary factors associated with hypertension. Nat Rev Cardiol. 2011;8(8):456-65. https://doi.org/10.1038/nrcardio.2011.75 
7. Goulart EL. Hipertensão na gestação. Porto Alegre; 2012.

8. Impey L, Greenwood C, Sheil O, MacQuillan K, Reynolds M, Redman C. The relation between pre-eclampsia at term and neonatal encephalopahty. Arch Dis Child. 2001; 85:170-2. https:// doi.org/10.1136/fn.85.3.F170

9. Ramakrishnan, U. Nutrition and low birth weight: from research to practice. Am J Clin Nutr. 2004;79:17-21.

10. Martins-Costa SH. Doença Hipertensiva na Gravidez. In: Accioly, E.Saunders C, Lacerda, EMA. Nutrição em Obstetrícia e Pediatria. Rio de Janeiro: Cultura médica; 2002. p. 189-207.

11. Godfrey K, Barker D. Fetal nutrition and adult disease. Am J ClinNutr. 2000;71:1344S-52S.

12. Ministério da Saúde (BR) Proporção de partos hospitalares. Brasília: Ministério da Saúde; 2010 [cited 2013 Jun 15]. Available from: http://tabnet.datasus.gov.br/cgi/deftohtm.exe?idb2012/f07.def

13. Padovani RM, Amaya-Farfán J, Colugnati, FAB, Domene SMÁ. Rev Nutr. 2006;19(6):741-60. https://doi.org/10.1590/S141552732006000600010

14. Epi Info (TM) [computer program] Version 3.4. Database and statistics software for public health professionals. Centers for Disease Control and Prevention (CDC); 2007.

15. Calderon IMP, Cecatti JG, Veja CEP. Intervenções benéficas no pré-natal para prevenção da mortalidade materna. Rev Bras
Ginecol Obstet. 2006;28(5):310-5. https://doi.org/10.1590/S010072032006000500008

16. Lacerda EMA, KG, Da Cunha CB, Leal MC. Consumo alimentar na gestação e no pós-parto segundo cor da pele no município do Rio de Janeiro. Rev Saúde Pública. 2007;41(6):985-94. https://doi. org/10.1590/S0034-89102007000600014

17. Andreto LM, De Souza Al, Figueiroa J N, Cabral-Filho J E. Fatores associados ao ganho ponderal excessivo em gestantes atendidas em um serviço público de pré-natal na cidade de Recife, Pernambuco, Brasil. Cad Saúde Pública. 2006;22 (11):2401-9. https://doi. org/10.1590/S0102-311X2006001100014

18. Konno SC, Benicio MHDA, Barros AJD. Fatores associados à evolução ponderal de gestantes: uma análise multinível. Rev Saúde Pública 2007;41(6):995-1002. https://doi.org/10.1590/S003489102007000600015

19. Burland L. Transferência condicionada de renda e segurança alimentar e nutricional. Ciênc Saúde Coletiva. 2007;12(6):1441-51. https://doi.org/10.1590/S1413-81232007000600007

20. Lima GSP, Sampaio HAC. Influência de fatores obstétricos, socioeconômicos e nutricionais da gestante sobre o peso do recémnascido: estudo realizado em uma maternidade em Teresina, Piauí. Rev Bras Saúde Matern Infant. 2004;4(30):253-61. https://doi. org/10.1590/s1519-38292004000300005 